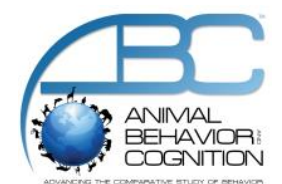

\title{
A Tool Use Task Proves Enriching for a Captive Sea Otter (Enhydra lutris)
}

\author{
Pepper R. Hanna ${ }^{1, *}$, Erin E. Frick ${ }^{1}$, \& Stan A. Kuczaj, II ${ }^{1}$
}

${ }^{1}$ University of Southern Mississippi

*Corresponding author (Email: pepper.hanna@eagles.usm.edu)

Citation - Hanna, P. R., Frick, E. E., \& Kuczaj, S. A. II. (2016). A tool use task proves enriching for a captive sea otter. Animal Behavior and Cognition, 3(2), 88-94. doi: 10.12966/abc.03.05.2016

\begin{abstract}
Environmental enrichment is used to improve an animal's physical and psychological well-being while housed in a captive environment. Alligood and Leighty (2015) suggested that enrichment that emulates the natural and preferred behaviors of the species may be optimal for improving welfare. Cognitive tasks that mirror challenges in an animal's natural environment may prove especially beneficial (Washburn, 2015). The present study was designed to determine whether two sea otters (Enhydra lutis), Emma and Buck, were capable of learning to use a novel tool in a novel context. The otters' time spent engaged with the apparatus was also examined to determine if such a task would be enriching to these animals. Although the sea otters were not successful in solving the tool use task, interaction with the apparatus appeared enriching to Emma. She spent on average 55.4\% of each trial at the apparatus, whereas Buck spent only 6.2\%. Only Emma's interaction with the apparatus suggests that this device was very interesting and consequently enriching for her. Cognitive challenges, then, may be enriching even when animals fail to solve the intended problem.
\end{abstract}

Keywords - Sea otter, Enhydra lutis, Enrichment, Tool use

Environmental enrichment has been used as a means of varying the environment of captive animals and increasing production of species-typical and goal-directed behaviors (Kuczaj, Lacinak, \& Turner, 1998; Shyne, 2006). Forms of environmental enrichment include opportunities for foraging, toys and other objects the animal can manipulate, changes to the physical environment, and visual or auditory stimuli (Brent \& Stone, 1996; Newberry, 1995). Many facilities implement enrichment strategies as a means of reducing stereotypic or abnormal behavior (Shyne, 2006). The success of these enrichment strategies is sometimes assessed as a function of the amount of decline observed in the rates of undesired behaviors (Shyne, 2006). It is also possible to use the amount of time the animal engages with or manipulates the device while it is available as a measure of the efficacy of the enrichment procedure (Brent \& Stone, 1996; Kuczaj, Lacinak, Otto, Trone, \& Solangi, 2002).

Not all methods of environmental enrichment are equally effective (Tarou \& Bashaw, 2007). Foraging tasks have been found to be a particularly successful means of environmental enrichment in diminishing stereotypic behaviors, and in encouraging participation in the task, with animals maintaining a high rate of interaction with foraging tasks over relatively long periods of time (Bayne et al., 1991, Boccia, 1989; Westergaard \& Frazaszy, 1985). In addition, Shyne (2006) found that when compared to changes in physical environment and the addition of scents, food puzzles provided more benefit in reducing the rates of stereotypic behaviors in a variety of species. 
The long-term benefits of an enrichment program can be affected by the specifics of implementation. Habituation is one concern with establishing a new enrichment program. Kuczaj et al. (2002) found that when a device is provided to an animal for relatively short, variable intervals, the effects of habituation are minimized. Charmoy, Sullivan, and Miller (2015) reported that use of automatic feeders that allow for the provision of food at random periods throughout the day decreased the predictability of a captive environment and led to an increase in the foraging behaviors of captive gorillas. In their comparative study on the effectiveness of an enrichment device for several species, Kuczaj et al. (2002) found that species differed in their interactions with a particular enrichment device, and that there were individual differences in these interactions as well. Alligood and Leighty (2015) suggested that enrichment could be most beneficial when it encourages a natural and preferred behavior of an animal. All of this suggests that it is important to consider the natural behavioral repertoire of the target species when determining possible enrichment devices. In addition, it is necessary to consider individual differences within the species as well. What is stimulating to one animal is not necessarily interesting to other members of the species.

Kuczaj et al. (1998) suggested that cognitive tasks can be important forms of enrichment, and the key to the enriching qualities of these tasks is the ability to create a moderately discrepant event. These are situations in which familiarity and novelty are balanced, so that a degree of familiarity allows the animal to interpret and deal with the novelty that makes the task interesting. Washburn (2015) noted that creating more difficult tasks in which the animal is challenged is an important component of enrichment devices. This suggested that the animal may benefit psychologically from successful completion of difficult tasks.

A tool use task is an ideal paradigm in which to investigate the efficacy of a cognitive task as enrichment for a captive sea otter due to the fact this is a species known to use tools in the wild. Specifically, they have been observed to rest rocks or stones on their stomach and bang mollusk shells on them until the shells can be opened (Fisher, 1939; Hall \& Schaller, 1964). The series of behaviors involved in this process has been described in several populations of sea otters, suggesting that this behavior is genetically transmitted within the species (Bentley-Condit \& Smith, 2010). This predisposition may enable sea otters to understand that an object can be used to obtain food, which may assist an otter in using a novel tool to obtain food in a novel task. The goal of the present study was to establish a moderately discrepant event that creates a stimulating task to motivate the otter, increasing both the likelihood of interaction and the enriching quality of the task.

\section{Method}

\section{Subjects}

The subjects used in this study included the two sea otters housed at the Audubon Aquarium in New Orleans, Louisiana. At the time of the study the male otter, Buck, was 16 years old and the female, Emma, was 15 years old. Both animals had previously stranded, and after rehabilitation were determined non-releasable by the National Marine Fisheries Service. The otters were housed together in a 25,000 gal. habitat with two separate pools of varying depths. The two pools were able to be closed off, granting access to one or both pools. A small holding pool was located behind the exhibit pools. Testing took place on a small platform beside the holding pool. Only the study animal was allowed access to the holding pool during trials. The second otter remained within the exhibit pool during trials. The otters' diet consisted of approximately $10 \mathrm{lbs}$. of seafood each day.

\section{Apparatus}

The apparatus was comprised of a cutting board with a PVC pipe pulley system attached to two hook-shaped tools (Figure 1). Each hook was identical in shape, size, weight, and color. This tool choice paradigm presented the test subject with the choice between the two tools that had food placed in a 
predetermined pattern around both tools. The food was positioned such that only one tool could be used to retrieve it. Pulling the other tool resulted in the correct tool being moved out of reach, necessitating selection of the correct tool as the first choice. In order to ensure the otters could only choose one tool for a given trial, the apparatus was presented via a gated wall that allowed their paws to reach through and interact with the apparatus, but did not allow full access. The experimental setup is depicted in Figure 2. Shrimp was used as a primary reinforcement for correct use of the tool. This was in addition to their typical meals during the day.

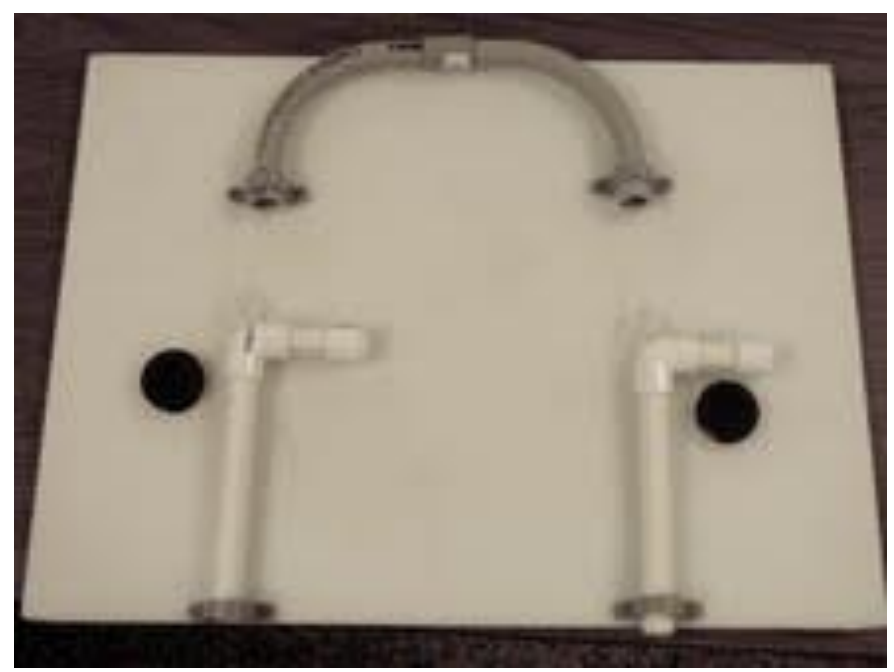

Figure 1. The apparatus layout.

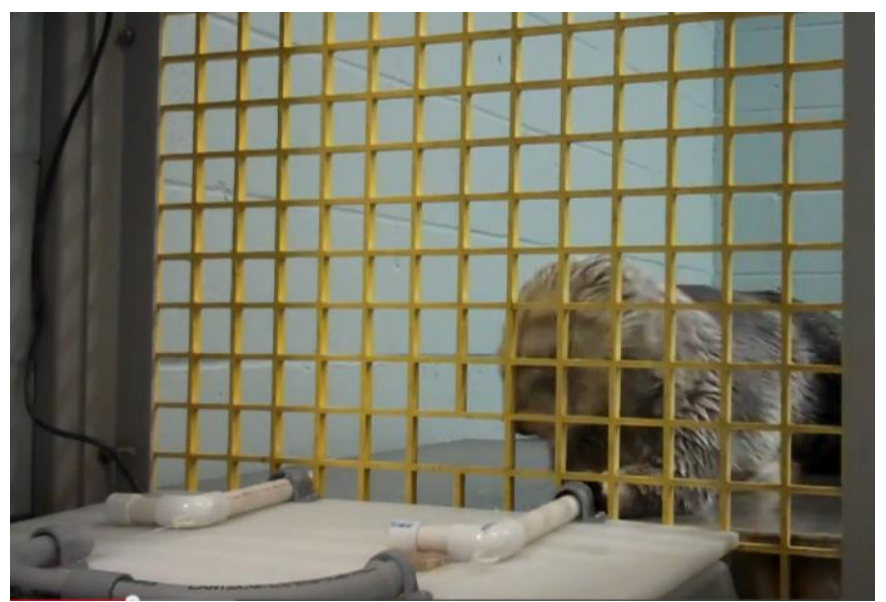

Figure 2. Example of otter interaction with the apparatus during a trial.

\section{Procedure}

There was no training or exposure to the apparatus prior to the experimental trials. A digital video camera (Flip Ultra Video Camera) was set up in advance to record all trials. All trials took place in the morning prior the first feeding period. Testing was done in the back holding room of the sea otter exhibit at the Audubon Aquarium in New Orleans. The apparatus was set up prior to the start of trials while the otters were kept in a different area. Trials began once the otters were allowed access into the holding room. Trial length varied between 2-10 min. Trial length varied in order to minimize opportunity of the otter to break pieces of the apparatus, while still maintaining the goal of the study to create an effective enrichment device. There were 10 possible arrangements of food and tool orientation presented to both 
otters. A total of 20 trials were conducted for Emma and 12 for Buck. Each otter was tested individually. Data analysis focused on time spent interacting with the apparatus and performance on the tool-choice task.

\section{Results}

\section{Tool Use Performance}

Emma performed at chance levels on the tool use task. She chose the correct tool on only 8 of the 20 trials (40\%). Buck participated in 12 trials. He only made a choice on the first four trials. He made a correct choice on three of these trials (25\% of all trials and $75 \%$ of trials with a choice). Due to this low rate of interaction with apparatus it is difficult to interpret these results.

\section{Interaction with the Apparatus}

Emma. On average, she spent $55.4 \%$ of each trial time manipulating the apparatus, pushing and pulling the tool through the gate barrier. On 3 of her 8 successful trials, although the food was within reach, she would continue to push or pull on the apparatus before consuming the food. Her delay in eating the shrimp ranged from $15 \mathrm{~s}$ to $24 \mathrm{~s}$. On 2 of her successful trials, her interaction with the apparatus caused the shrimp to fall from the cutting board and out of reach. This did not result in a decline in her interaction rate with the apparatus. Her interaction rates with the apparatus during these trials were $75.5 \%$ and $72.1 \%$, greater than the average time she spent with the apparatus. During the 5th trial, she was able to break off a piece of the PVC pipe. Once removed, she took this piece into the exhibit with her and did not return to the apparatus for the rest of the trial. This appeared to have an effect on the amount of time she spent with the apparatus during subsequent trials. In the trials preceding this event, she spent an average of $40.6 \%$ of the trial time engaged with the apparatus. During the trials following this event she spent an average of $80.5 \%$ of the trial time with the apparatus.

Buck. Buck spent an average of $6.2 \%$ of the trial time at the apparatus. This includes four trials in which Buck never approached the apparatus. The average time spent at the apparatus for trials in which he did approach the apparatus was $9.2 \%$. The trials in which Buck spent the greatest amount of time at the apparatus occurred in the first four trials. He spent an average of $12.9 \%$ of these initial trials at the apparatus. After this point Buck typically spent a few seconds at the apparatus and did not make any attempts to use the tools to obtain the food.

\section{Possible Spontaneous Tool Use Event}

On one occasion, Emma pushed both tools out of her reach. Unable to reach either tool, Emma left the experimental apparatus and swam to her exhibit pool. She then returned to the apparatus carrying a plastic toy tire in her forepaw. She proceeded to hit this object against the gate barrier separating her from the apparatus. This behavior continued for several seconds. Emma's behavior during this incident was consistent with the previously suggested definitions of tool use (e.g., Mann \& Patterson, 2013) with her goal being to obtain access to the apparatus.

\section{Discussion}

Enrichment should provide rewarding stimulation for the behavioral and psychological needs of captive animals, as well as help animals emulate the species-specific behaviors of their wild conspecifics. Effective enrichment may be implemented through the use of novel objects, tactile stimulation, food variation, cognitive tasks, and social interactions with conspecifics or other species (Hutchins, Hancock, \& Crockett, 1984; Kreger, Hutchins, \& Fascione, 1998). Individual differences affect the efficacy of enrichment (Kuczaj et al., 2002), as was the case in this study. Buck did not find the apparatus to be 
enriching or rewarding, but Emma chose to interact with the apparatus on numerous occasions. Charmoy et al. (2015) found that male gorillas' foraging behavior was impacted less by an enrichment device than was that of female gorillas. However, Eskelinen, Winship, and Borger-Turner (2015) found that male dolphins were more likely to interact with an enrichment device than were female dolphins. Since there were only two animals involved in this study it is unclear if the differences between Buck's and Emma's interaction rates represent individual or sex differences.

The enrichment usage hypothesis emphasizes increased frequency of object use and speciesspecific behaviors as a measure of the effectiveness of a given form of enrichment (Lutz \& Novak, 2005). In this view, increased object manipulation and increased active behaviors when enrichment is present, serve as indicators that the object or task presented was enriching (Swaisgood et al., 2001). In the present study, Buck's lack of interest in interacting with the apparatus indicated this was not enriching for him. In contrast, Emma appeared to find the task highly enriching, albeit not for the reasons anticipated. Once Emma was able to break off a piece of the PVC pipe, her interactions with the apparatus did not appear to be motivated by using the tools to obtain food, but instead appeared to be aimed toward interacting with the apparatus until more pieces were obtained. Our efforts to prevent this resulted in a more "otter-proof" apparatus, which in turn resulted in Emma spending more time attempting to thwart our efforts.

Emma did show some interest in the food reinforcement, yet even when she had chosen the "correct" tool she would delay the consumption of the food for several seconds while continuing to interact with the apparatus. In addition, Emma spent more time with the apparatus as her experience with it increased. The fact that the amount of time interacting with the apparatus increased over trials supports the notion that interaction with the apparatus was enriching.

The intervals of time where Emma could interact with the apparatus varied across trials. Kuczaj et al. $(1998,2002)$ demonstrated that limiting and varying the amount of time an animal has access to a form of enrichment decreases the likelihood of the animal habituating to the object, and so maintains the novelty of the object for longer periods of time. Such schedule variability was used in this study, and may have increased the novelty of the apparatus as an enrichment device.

In the isolated instance when Emma pushed both of the tool choices out of reach and she could no longer interact with the apparatus, that form of enrichment was no longer available to her. The removal of the enriching stimulus (the apparatus) may have frustrated Emma. In animals, frustration occurs when the animal is unable to do something that they are highly motivated to complete, and motivation arises from wanting to engage in a behavior that is prevented (Dawkins, 1990). Emma had deprived herself of interaction with the apparatus, and the longer this deprivation persisted, the higher the motivation to alleviate the resulting stress from frustration. This serves as one possible explanation for why she was motivated to find some way of regaining access to the apparatus; in this case by attempting to "knock down" or "break through" the barrier with a plastic tire. However, it is possible for other variables to have influenced this event.

The behavior Emma appeared to use to attempt to remove the gate barrier was also consistent with the definition of spontaneous tool use: an untrained and unsolicited behavior that involves manipulating an object to purposefully achieve a goal (Mann \& Patterson, 2013). In this instance, the "goal" would appear to be the removal of the barrier to the apparatus, as interaction with the apparatus was a strong form of enrichment for Emma. Her use of the plastic tire to repeatedly hit the gate fits the definition of "purposely manipulating an object." Of course, it is possible that she was hitting the gate with the tire because she was frustrated rather than because she wished to hammer an opening through the gate.

Foraging for food is a common natural behavior in the sea otter, but such behaviors are not typically necessary in captivity. The use of foraging devices as a form of enrichment can increase the activity level of the animals, and may promote species-specific behaviors (Celli, Tomonaga, Udono, Teramoto, \& Nagano, 2003). The type of device used for enrichment does not need to mimic foraging in the wild in order to promote foraging-behaviors (Lutz \& Novak, 2005). Rather, the task needs to stimulate the process of working for food, and can vary in its complexity based upon the species and individual differences in aptitude or skill (Heath, Shimoji, Tumanguil, \& Crockett, 1992). Species that utilize tools 
during their foraging, such as the sea otter, should see an increase in species-specific behaviors when presented with a tool-use task in a captive setting. Celli et al. (2003) introduced a variety of objects that could be utilized as tools for honey fishing in chimpanzees, a form of foraging found in wild populations of chimpanzees. This resulted in increases in activity budget for foraging behaviors. In the present study, the tool-use task did not represent a naturally occurring situation that a sea otter would encounter in the wild. However, the forced choice paradigm presented to the otters required them to solve a problem to obtain a food reward utilizing the tool objects available. Environmental enrichment sometimes encourages behaviors that are indicative of the species natural repertoire (Kuczaj et al., 2002) and such was observed during Emma's interactions with the apparatus. Emma produced behaviors consistent with those of wild sea otters' foraging behaviors, such as banging pieces of the apparatus against the wall and using the tire as a tool to attempt to break down the barrier that prevented her from interacting with the apparatus. The tool-use task appeared to be enriching for Emma, and adds to the literature that demonstrates that cognitive tasks may be used as forms of environmental enrichment (e.g., Clark, Davies, Madigan, Warner, \& Kuczaj, 2013). Future research should focus on the interaction of individual differences and cognitive task type to better determine the enriching qualities of cognitive tasks. A larger sample size could also help to clarify the role of gender in determining responses to an enrichment device.

\section{Acknowledgements}

The authors would like to thank Suzanne Smith and all the staff at The Audubon Aquarium of the Americas for their cooperation and assistance on this project. We wish to thank Nicole Hardy and Kristin Ritch for their help with data collection.

\section{References}

Alligood, C., \& Leighty, K. (2015). Putting the "E" in SPIDER: Evolving trends in the evaluation of environmental enrichment efficacy in zoological settings. Animal Behavior and Cognition, 2, 105-123.

Bayne, K., Mainzer, H., Dexter, S., Campbell, G., Yamada, F., \& Suomi, S. (1991). The reduction of abnormal behaviors in individually housed rhesus monkeys (Macaca mulatta) with a foraging/grooming board. American Journal of Primatology, 23, 23-35.

Bentley-Condit, V. K., \& Smith, E. O. (2010). Animal tool use: Current definitions and an updated comprehensive catalog. Behaviour, 147, 185 - 232.

Brent, L. \& Stone, A. (1996). Long-term use of televisions, balls, and mirrors as enrichment for paired and singly caged chimpanzees. American Journal of Primatology, 39, 139-145.

Boccia, M. L. (1989). Long-term effects of a natural foraging task on aggression and stereotypies in socially housed pigtail macaques. Laboratory Primate Newsletter, 28, 18-19.

Celli, M. L., Tomonaga, M., Udono, T., Teramoto, M., \& Nagano, K. (2003). Tool use task as environmental enrichment for captive chimpanzees. Applied Animal Behaviour Science, 81, 171-182.

Charmoy, K., Sullivan, T., \& Miller, L. J. (2015). Impact of different forms of environmental enrichment on foraging and activity levels in gorillas (Gorilla gorilla gorilla). Animal Behavior and Cognition, 2, $233-$ 240.

Clark, F. E., Davies, S. L., Madigan, A. W., Warner, A.J., \& Kuczaj, S.A. II (2013). Cognitive enrichment for bottlenose dolphins (Tursiops truncatus): Evaluation of a novel underwater maze device. Zoo Biology, 32, $608-619$.

Dawkins, M. S. (1990). From an animal's point of view: Motivation, fitness, and animal welfare. Behavioral and Brain Sciences, 13, 1-9.

Eskelinen, H. C., Winship, K. A., \& Borger-Turner, J. (2015). Sex, age, and individual differences in bottlenose dolphins (Tursiops truncatus) in response to environmental enrichment. Animal Behavior and Cognition, 2, $241-253$.

Fisher, E. M. (1939). Habits of the southern sea otter. Journal of Mammology, 20, 21 - 36.

Hall, K. R. L., \& Schaller, G. B. (1964). Tool-using behavior of the California sea otter. Journal of Mammalogy, 45, 287-298. 
Heath, S., Shimoji, M., Tumanguil, J., \& Crockett, C. (1992). Peanut puzzle solvers quickly demonstrate aptitude. Lab Primate Newsletter, 31, 2-13.

Hutchins, M., Hancocks, D., \& Crockett, C. (1984). Natural solutions to the behavioral problems of captive animals. Zoologische Garten, 54, 28-42.

Kreger, M. D., Hutchins, M., \& Fascione, N. (1998). Context, ethics, and environmental enrichment in zoos and aquariums. In D. M. Shepherdson, J. D. Mellen, \& M. Hutchins (Eds.), Second nature: Environmental enrichment for captive animals (pp. 59-82). Washington, DC: Smithsonian Institution Press.

Kuczaj, S.A. II, Lacinak, T., Otto, F., Trone, M., \& Solangi, M. (2002). Keeping environmental enrichment enriching. International Journal of Comparative Psychology, 15, 127 - 137.

Kuczaj, S.A. II, Lacinak, T., \& Turner, T. (1998). Environmental enrichment for marine mammals at Sea World. In D. M. Shepherdson, J. D. Mellen, \& M. Hutchins (Eds.), Second nature: Environmental enrichment for captive animals (pp. 314-328). Washington, DC: Smithsonian Institution Press.

Lutz, C. K., \& Novak, M. A. (2005). Environmental enrichment for nonhuman primates: Theory and application. Ilar Journal, 46, 178-191.

Mann, J., \& Patterson, E. M. (2013). Tool use by aquatic animals. Philosophical Transactions of the Royal Society B: Biological Sciences, 368, 1 - 11.

Newberry, R.C. (1995). Environmental enrichment: Increasing the biological relevance of captive environments. Applied Animal Behavior Science, 44, 229 - 243.

Shyne, A. (2006). Meta-analytic review of the effects of enrichment on stereotypic behavior in zoo mammals. Zoo Biology, 25, $317-337$.

Swaisgood, R. R., White, A. M., Zhou, X., Zhang, H., Zhang, G., Wei, R., Hare, V., Tepper, E., \& Lindburg, D. G. (2001). A quantitative assessment of the efficacy of an environmental enrichment program for giant pandas. Animal Behaviour, 61, 447-457.

Tarou, L. R., \& Bashaw, M. J. (2007). Maximizing the effectiveness of environmental enrichment: Suggestions from the experimental analysis of behavior. Applied Animal Behavior Science, 102, 189 - 204.

Washburn, D. (2015). The four Cs of psychological wellbeing: Lessons from three decades of computer-based environmental enrichment. Animal Behavior and Cognition, 3, 218 - 232.

Westergaard G. C., \& Fragaszy, D. M. (1985). Effects of manipulable objects on the activity of captive capuchin monkeys (Cebus apella). Zoo Biology, 4, 317-327. 